\title{
Domino Effect Analysis, Assessment and Prevention in Process Industries
}

\author{
Jun WU \\ School of Economics and Management, Beijing University of Chemical Technology, Beijing 100029, \\ China \\ E-mail:wujun@mail.buct.edu.cn \\ Hui YANG \\ School of Economics and Management, Beijing University of Chemical Technology, Beijing 100029, \\ China \\ E-mail: 18810941646@163.com \\ Yuan CHENG \\ Department of Automation, Tsinghua University, Beijing 100084, China \\ E-mail: qazcy1983@163.com
}

\begin{abstract}
Domino effect is a fairly common phenomenon in process industry accidents, which makes many process industry accidents serious and the consequent losses enhanced. Domino effect of the major accidents in chemical cluster is emphasized. Many researchers have studied domino effect in chemical clusters from different perspectives. In the review, we summarize the research from three aspects: The statistical analysis of domino accidents in chemical process industry, the evaluation of domino accidents and the prevention of domino accidents in chemical clusters by game theory. From the analysis, we can find the characteristic of domino accidents such as the time and the location, the origin and causes of domino accidents. The methods of assessing domino effects such as quantitative risk assessment (QRA), Bayesian networks (BN) and Monte Carlo simulation (MCS) are analyzed. The prevention of domino accidents in chemical clusters using game theory is seldom, and there is still much space for improvement in enterprises' efforts to prevent risk of domino accidents.
\end{abstract}

Keywords domino effect; quantitative assessment; game theory; chemical cluster

\section{Introduction}

The importance of domino effect on the occurrence and severity of the major accidents that take place in the process industry is emphasized. Accidents involving several plants do occur, and potential economic loss is often many times greater than that of single plant escalation accidents. In the book "Domino effects in the process industries: Modeling, prevention

Received June 10, 2015, accepted July 29, 2015

Supported by the National Natural Science Foundation of China (71372195, 71571010), Fundamental Research Funds for the Central Universities in BUCT, BUCT Fund for Disciplines Construction and Development (XK1522). Wu was supported by Key Scientific Research Base of Beijing University of Chemical Technology, State Administration for Cultural Heritage 
and managing", Reniers and Cozzani analyzed the main features of domino accidents ${ }^{[1]}$. The complexity of domino effect accident scenarios and many ways through which the escalation and propagation of accidents can take place were analyzed in this book. Although various researchers have provided suggestions, there is no generally accepted definition of what constitutes domino effects in the context of accidents in the chemical processing industry. Kadri showed an overview of some definitions identified with a review of the relevant documents ${ }^{[2]}$.

Domino effect is the most dangerous accidents that can happen within chemical clusters, a term by which the potential for a knock-on interaction between groups of installations in the event of an accident at one of the installations is connoted. According to Delvosalle ${ }^{[3]}$, a domino accident can be defined as a cascade of events in which the consequences of a previous accident are increased both spatially and temporally by the following ones, thus leading to a major accident. Domino accidents have brought about catastrophic accidents that took place in the chemical and process industries. A large proportion of domino accidents, i.e., $89 \%$ domino accidents are caused by flammable substances and petroleum products, which are main materials in the chemical industry ${ }^{[4]}$.

Many researchers have studied domino effect in chemical clusters from different perspectives.

Some focus on statistical analysis of domino accidents in the chemical process industry ${ }^{[5-7]}$, and others evaluate domino effect in chemical clusters ${ }^{[1,8]}$. In addition, there are studies on preventing domino accidents in chemical clusters by using game theory ${ }^{[9,10]}$. Though the significance of domino accidents in chemical clusters is well recognized, there is much space for improvement in enterprises' efforts to prevent the risk of domino accidents.

In our work, we summarize the research from three aspects: The statistical analysis of domino accidents in the chemical process industry, the evaluation of domino accidents and the prevention of domino accidents in chemical clusters by using game theory. In statistical analysis, we analyze the characteristics of domino accidents, such as the time and the location, the origin and causes of domino accidents. In the evaluation of domino accidents, we study the methods of assessing domino effect on quantitative risk assessment (QRA), Bayesian networks $(\mathrm{BN})$, and Monte Carlo simulation (MCS). In the prevention of domino accidents, we state the evolution of the game theory of preventing domino effect.

The rest of the paper is organized as follows. The next section theoretically review the research on the analysis of past accidents. In Section 3 we provide a specific framework of the quantitative assessment of domino scenarios. We analyze the prevention of domino accidents by using game theory in Section 4. We summarize the research and suggest future research topics in the last section.

\section{Domino Accidents Analysis}

\subsection{Survey of Domino Accidents}

Domino accidents can be analyzed from research literature, reports of certain institutions and appropriate databases such as Major Hazard Incident Data Service (MHIDS) database, Major Accidents Reporting System (MARS) and Major Accident Hazards Bureau (MAHB). Some researchers analyze diverse historical surveys performed on domino accidents and main characteristics of this phenomenon. We will describe the survey of domino accidents from two 
aspects. The one is the research which only includes domino accidents, and the another is the research which involves domino accidents.

\subsubsection{Only Domino Accidents}

There are some research with domino accidents in industrial plants or transportation. Abdolhamidzadeh et al. ${ }^{[11]}$ presented 73 domino accidents that occurred in industrial plants and transportation from 1917 to 2008, and found that $21 \%$ of accidents involving domino effect had occurred in transportation. The substances involved, the level of domino effect and the impact on the population were analyzed. A small number of accidents (including accidents involving conventional explosives) were published and the ratio of first-level and second-level domino sequences was obtained. Moreover, Darbra et al. ${ }^{[6]}$ collected 225 domino accidents that happened in process/storage plants and the transportation of hazardous materials from 1961 and 2007. The location of accident, the type of accident, the materials involved, the causes and consequences and the most common accident sequences were analyzed. The analysis of installation and operation types showed that the most critical area was storage (35\%), followed by process plants $(28 \%)$ and transportation $(19 \%)$. The most frequent causes were external events $(31 \%)$, followed by mechanical failures $(29 \%)$ and human factor $(28.9 \%)$. Flammable substances involved in $89 \%$ were the most frequently found accidents in domino accidents. Furthermore, Abdolhamidzadeh et al. ${ }^{[12]}$ studied 224 major process industry accidents involving domino effect from 1917 to 2009. Most of accidents had occurred in process plants, followed by transportation. The location, the type of plant, substance involved, sequence of accidents, deaths, injuries, other losses, and the source of accidents were analyzed. It can be found that $72 \%$ of domino accidents happened in the developed countries and the rest of domino accidents happened in the developing countries (28\%). Among others aspects, the type of materials involved, the primary events, the contribution of transportation and fixed plants, the frequencies of the accident sequence lengths and the number of fatalities were also analyzed and the corresponding conclusions were reached.

Other, Delvosalle et al. ${ }^{[3]}$ studied 41 domino accidents that happened from 1944 to 1995. Among these accidents, $66 \%$ happened to the last 20 years of the period. In view of the type and the essence of primary and secondary accidents, the types of domino effect were classified. Chen et al. ${ }^{[13]}$ published 318 domino accidents from 1951 to 2012 and analyzed to get their main factors, such as distribution of accidents, causes, substances involved and domino sequences. The statistics showed that most of domino accidents (71.1\%) occurred in the developed countries and $28.9 \%$ of accidents occurred in the developing countries. Human failure was most likely to cause an accident in China, compared with the developed countries. The most of accidents were caused by flammable substances, and the most frequent secondary/tertiary domino sequence was due to explosion $(24.8 \%)$.

\subsubsection{Involving Domino Accidents}

A set of research is retrieved from literature and classified with regard to the substance involved and whether domino effects are present. Some researchers have studied accidents in- 
volving domino effect in industrial plants or transportation. Oggero et al. ${ }^{[14]}$ obtained 1932 accidents that occurred during the transport of hazardous substances by road and rail from the beginning of the 20th century to 2004. According to statistic, it showed that the frequency of accidents had a increasing trend and more than half of the accidents related to roads (63\%). The frequent of accidents, causes of accidents, the type of substance involved and the consequences were analyzed. Hemmatian et al. ${ }^{[15]}$ performed a historical survey of 330 accidents involving domino effect occurred in process/storage plants and in the transportation of hazardous materials from 1961 to 2013. The statistic showed that the most common accidents happened in process plants (38.5\%) and storage areas (33\%), followed by transfer operations (10.6\%). Further, the accidents occurred in the 21st century were obtained and compared with the total accidents. It can be seen that the most important causes of the domino accidents were mechanical failure, external events and human factor. When studying accidents occurred in the 21 st century, it was found that the proportion of human factor increased from $24.6 \%$ to $35 \%$.

Other, Kourniotis et al. ${ }^{[5]}$ presented 207 chemical accidents between 1960 and 1998 from the literature, competent authorities reports (CEPPO, HSE) and well established accident databases (MARS). Accidents were classified with regard to the substance involved and the existence of domino effect, and the characteristics of chemical accidents were analyzed. Ronza et al. ${ }^{[16]}$ studied 828 accidents happened in port areas from a database and identified the sequences of accidents. Considering the location of accidents, $40 \%$ occurred in the sea, and $21 \%$ did on land. It was worth noting that the remaining $39 \%$ occurred at a sea-land interface, which is a remarkable characteristic of ports areas. Gomez-Mares et al. ${ }^{[17]}$ made 84 accidental scenarios involving jet fires from several accidents databases, and showed that $50 \%$ of reported cases involving a jet fire caused primary event of a domino sequence. The origin of accidents, the type of substance involved, causes of accidents, the type of accidents and the consequences were analyzed. Zhang and Zheng ${ }^{[18]}$ analyzed 1632 hazardous chemical accidents (HCAs) occurring in China from 2006 to 2010 by the data from official sources. With analysis, they obtained the following characteristic of accidents, such as time volatility, location distribution, fixed facility type versus transportation type, injury versus death and causes of domino accidents.

\subsection{Characteristic of Domino Accidents}

In this section, the main features of accidents in which domino effect has taken place are analyzed, e.g. the time and location of domino accidents, the origin and causes of domino accidents.

\subsubsection{Distribution of Accidents According to Time and Location}

Concerning the evolution as a function of time, some researchers analyzed domino effect. Kourniotis et al. ${ }^{[5]}$ obtained 207 major chemical accidents occurred from 1961 to 2000, while majority of them occurred within the last two decades. Oggero et al. ${ }^{[14]}$ studied 1932 accidents that occurred during the transport of hazardous substances by road and rail from 1931 to 2005, and analyzed variations in the frequency of accidents as a function of time for chemical plants. It can be observed that there is a significant increase in the number of accidents in 
1981-1990 and 1990-2000. Darbra et al. ${ }^{[6]}$ analyzed domino accidents from 1961 to 2007, and found that the number of accidents increased in 1970-1980 and accident rate decreased since 1990. The decreasing accident rate since 1990 could be explained by general improvements in the safety culture of the chemical industry brought about by strict new regulations and more effective operator training. Chen et al. ${ }^{[13]}$ collected 318 domino accidents from 1951 to 2012 and analyzed chronological distribution of domino accidents. According to statistic, it indicated that main accidents was in the period from 2001 to 2012 . Hemmatian et al. ${ }^{[15]}$ plotted accidents from 1961 to 2013, and showed that the highest percentage of accidents (23.9\%) happened in the 1970's. The accident rate decreased in the 1990's, followed by the frequency increasing in the first decade of the 21st century. The frequency of domino accidents is summarized in Table 1. We can draw that the highest percentage of accidents is in 1971-1980 and 1981-1990. The chemical industry has grown continuously since the early 1970s. More and larger process plants and storage areas have been built, leading to an increasing of accidents.

Table 1 Frequency of occurrence of domino effect

\begin{tabular}{cccccc}
\hline Source & up to 1970 & $1971-1980$ & $1981-1990$ & $1990-2000$ & 2000-2013 \\
\hline Kourniotis et al. $^{[5]}$ & 8.2 & 20.3 & 35.7 & 35.7 & \\
Oggero et al. ${ }^{[14]}$ & 4.2 & 11.55 & 28.43 & 43.14 & 12.68 \\
Darbra et al. $^{[6]}$ & 22 & 31 & 28 & 11 & 8 \\
Chen et al. $^{[13]}$ & 20 & 22 & 21 & 10 & 27 \\
Hemmatian et al. $^{[15]}$ & 16.7 & 23.9 & 22.4 & 12.7 & 24.2 \\
\hline
\end{tabular}

As the main characteristic of process industry, the location of domino accidents was also studied, which had a significant effect on the occurrence and severity of accidents, can change from one country to another. The accidents were divided into three groups depending on the country where they had occurred by applying both political and development-based criteria. The three main groups were the European Union, other developed countries including Australia, Canada, Japan, New Zealand, Switzerland, Norway, the United States, and the rest of the world.

Kourniotis et al. ${ }^{[5]}$ derived 207 major chemical accidents of the developed countries from relevant literature, and showed that the most of accidents happened in the Europe. Darbra et al. ${ }^{[6]}$ studied 225 accidents involving domino effect and found that more than $80 \%$ of accidents involving domino effect occurred in the developed countries. They divided accidents into three categories according to the country in which they occurred: The European Union (25\%), other developed countries (56\%) and the rest of the world (19\%). Hemmatian et al. ${ }^{[15]}$ performed on 330 accidents involving domino effect occurred in process/storage plants and in the transportation of hazardous materials, and classified the accidents in three main groups depending on the country where they had occurred: The European Union (21.8\%), other developed countries $(54.5 \%)$ and the rest of the world $(23.7 \%)$. By this classification, it can be seen that $76.3 \%$ of domino accidents occurred in the developed countries. Hemmatian et al. studied the distribution of domino accidents in the 21st century, and found that $14.3 \%$ accidents were corresponded to the EU, $44 \%$ to "other developed countries" and $41.7 \%$ to the rest of the world. Comparing two sets of results, it can be concluded that the frequency of domino accidents has decreased in the developed countries, while an increasing trend in the rest of countries. 
Table 2 Location of occurrence of domino effect

\begin{tabular}{cccc}
\hline Source & Europe & other developed countries & the rest of the world \\
\hline Kourniotis et al. ${ }^{[5]}$ & 61.4 & 38.6 & 19 \\
Darbra et al. ${ }^{[6]}$ & 25 & 56 & 23.7 \\
Hemmatian et al. ${ }^{[15]}$ & 21.8 & 54.5 & \\
\hline
\end{tabular}

The analysis showed that the developing countries had an increasing safety problem with their chemical industry. The location of domino accidents is summarized in Table 2. We can see that the highest frequency of domino accidents is in the developed countries. With distribution of domino accidents in 21st century, we can draw that the frequency of domino accidents has decreased in the developed countries, while an increasing trend in the rest of countries. A main conclusion obtained by the survey is that the frequency and the severity of the accidents decrease through the efforts devoted in the industrialized countries to improve the safety of both process plants and transportation of hazardous materials. While, going with development of economy, the frequency of domino accidents increases in the developing countries. Therefore, a similar effort should be applied urgently to the developing countries to improve safety of both process plants and transportation of hazardous materials.

\subsubsection{Origin and Causes of Domino Effect}

Domino effect is common phenomenon both in fixed plants and in transportation. Some researchers have given data on the distribution between these two possibilities. There are some research with the two aspects. Ronza et al. ${ }^{[16]}$ selected 828 accidents in port areas from a database, which had been used to identify the sequences of accidents. It was found that $7 \%$ of accidents involving domino effect had occurred in transportation. Transfer essentially loading/unloading accounted for $34 \%$ of cases. Thus, transport and transfer occupied $41 \%$. Fixed plants made up $59 \%$ (12\% storage, $2 \%$ process and $35 \%$ other). Darbra et al. ${ }^{[6]}$ analyzed 225 accidents, and obtained that $18.7 \%$ of cases had happened in transportation, whereas transfer essentially loading/unloading accounted for $13.3 \%$. Thus, transport and transfer occupied $32 \%$. Fixed plants made up $68 \%$ (35\% storage, $28 \%$ process and $5 \%$ other). Abdolhamidzadeh et al. ${ }^{[12]}$ selected 224 accidents, and found that accidents involving domino effect occurred in transportation accounted for $20 \%$ and the remaining of $80 \%$ occurred in fixed installations. They obtained the distribution among the diverse transport modes: $40 \%$ road, 39\% rail, $13 \%$ shipping and $8 \%$ pipeline. Chen et al. ${ }^{[13]}$ collected and analyzed 318 domino accidents from 1951 to 2012 to get their main factors, such as distribution of accidents, causes, involved substances and domino sequences. The statistic showed that transport and transfer accounted for $24.5 \%$. Fixed plants made up $75.5 \%$ (41.8\% storage, $33.7 \%$ process). Hemmatian et al. ${ }^{[15]}$ performed 330 accidents involving domino effect occurred in process/storage plants and in the transportation of hazardous materials. The statistic showed that transport and transfer occupied $24.4 \%$. Fixed plants made up $75.6 \%$ (34.6\% storage, $34.6 \%$ process and $6.4 \%$ others). Hemmatian et al. also analyzed those accidents occurred recently of the 21 st century from 2000 to 2013, and found that transport and transfer accounted for $14.3 \%$. Fixed plants made up $85.7 \%$ ( $17.4 \%$ storage, $51.2 \%$ process and $8.1 \%$ others). The origins of domino effect are 
summarized in Table 3. It is clear that domino accidents more occurred in fixed installation than transportation. Accidents occurred in transfer operations continue having a constant and important occurrence. Equipment safety devices and methods of loading/unloading should be improved, as well as the specific training provided to the operators. The efforts devoted to improve the safety of equipment and methods of loading/unloading are necessary.

Table 3 Origin of domino accidents

\begin{tabular}{clc}
\hline Source & transportation initiated & fixed installation \\
\hline Ronza et al. ${ }^{[16]}$ & 41 & 59 \\
Darbra et al. ${ }^{[6]}$ & 32 & 68 \\
${\text { Abdolhamidzadeh et al. }{ }^{[12]}}$ & 20 & 80 \\
Chen et al. ${ }^{[13]}$ & 24.5 & 75.5 \\
Hemmatian et al. ${ }^{[15]}$ & 24.4 & 75.6 \\
\hline
\end{tabular}

Some researchers studied the causes of the primary accident in detail in the survey. According to the categories established in the Major Hazardous Incident Data Service (MHIDAS) database, they classified the causes into external events, mechanical failure, human error, impact failure, violent reaction (runaway reaction), instrument failure, upset process conditions and services failure. Because some accidents were triggered by more than one generic cause, the percentages sum up to more than 100.

In Gomez-Mares et al. ${ }^{[17]}$, 37\% accidents analyzed were caused by mechanical failure, in which coupling or flange leakage was the main specific cause. The human factor accounting for $29 \%$ of the cases was the second general cause of accidents, followed by impact failure $(27 \%)$, external events (19\%), and all remaining causes (7\%). The general cause was unknown for $17 \%$ of the cases. Darbra et al. ${ }^{[6]}$ found that external events (31\%) and mechanical failure (29\%) were the main causes. It can be seen that $21 \%$ of the accidents were caused by human error, which is a similar value to the one obtained by Vílchez et al., who found that $24 \%$ of accidents analyzed in fixed installations and in transportation occurred by human error. Hemmatian et al. ${ }^{[15]}$ showed that mechanical failure $(35.2 \%)$ and external events $(29.4 \%)$ were the main causes of accidents. Human error caused $24.6 \%$ of accidents. These values have increased with respect to those of Darbra et al. ${ }^{[6]}$. Hemmatian et al. analyzed general causes of domino accidents in the 21st century, and showed the main general causes. As in the general study, there were significant contributions also from mechanical failure and human factor in the 21st century. The proportion of mechanical failure increased from $35.2 \%$ to $40 \%$, accompanied by the proportion of human factor increasing from $24.6 \%$ to $35 \%$. The generic causes that initiated a domino accident in the cases included in this analysis are summarized in Table 4 . We can know that mechanical failure is the main cause of domino effect, followed by human error and external events. Design, installation and maintenance of machines should be stressed to decrease domino effect. Human factor has a very important influence on domino accidents. Therefore, the training of operators, both in maintenance and plant operation, should be significantly improved particularly on the developing countries. 
Table 4 General causes of primary accidents

\begin{tabular}{|c|c|c|c|c|c|c|c|c|}
\hline Source & $\begin{array}{l}\text { mechanical } \\
\text { failure }\end{array}$ & $\begin{array}{l}\text { human } \\
\text { factor }\end{array}$ & $\begin{array}{l}\text { external } \\
\text { events }\end{array}$ & $\begin{array}{l}\text { impact } \\
\text { failure }\end{array}$ & $\begin{array}{l}\text { violent } \\
\text { reac- } \\
\text { tion }\end{array}$ & $\begin{array}{l}\text { instrument } \\
\text { failure }\end{array}$ & $\begin{array}{l}\text { upset } \\
\text { pro- } \\
\text { cess }\end{array}$ & others \\
\hline $\begin{array}{l}\text { Gomez-Mares } \\
\text { et al. }{ }^{[17]}\end{array}$ & 37 & 29 & 19 & 27 & 2 & 4 & 1 & 17 \\
\hline Darbra et al. ${ }^{[6]}$ & 28.9 & 20.9 & 30.7 & 17.8 & 9.3 & 3.6 & 2.2 & 1.2 \\
\hline $\begin{array}{l}\text { Abdolhamidzadeh } \\
\text { et al. }{ }^{[12]}\end{array}$ & 35.2 & 24.6 & 29.4 & 16.7 & 8.5 & 4.4 & 3.1 & 1.7 \\
\hline
\end{tabular}

\subsection{Summary for Domino Effect}

According to the data analyzed in the historical survey, we can obtain the results as follows. The maximum frequency of accidents is among 1971-1980 and 1981-1990. More and larger process plants and storage areas have been built and the chemical industry has grown continuously since the early 1960s. The increasing number of process plants leads to an increase of domino accidents. The highest frequency of domino accidents is in the developed countries before the 21st century. We can find that domino accidents have decreased in the developed countries, while an increasing trend in the developing countries by the distribution of domino accidents in the 21st century. It indicates the importance of safety culture measures in the developing countries. We should strive to improve equipment safety devices and methods of loading/unloading, as well as the specific training provided to the operators. It is necessary to enhance safety of equipment and methods of loading/unloading. The main cause of domino effect is mechanical failure, followed by human error and external events. In order to decrease domino effect, design, installation and maintenance of machines should be stressed. Human factor has a considerable influence in domino accidents. Therefore, the training of operators, both in maintenance and plant operation, should be significantly improved particularly in the developing countries.

\section{Quantitative Assessment of Domino Accidents}

Over the last decades, the risk of domino effect in chemical infrastructures has been recognized and considered in risk analysis, safety assessment and vulnerability analysis of chemical process plants ${ }^{[19-31]}$. All domino effect models share common aspects, and differences between them typically involve the way the accident sequences developed in the models or values used to assess equipment failure. The research of domino effect has primarily focused on quantitative risk assessment (QRA), Bayesian networks (BN) and Monte Carlo simulation (MCS). The next section will introduce the methods of assessing domino effect.

\subsection{Quantitative Risk Assessment}

\subsubsection{Quantitative Risk Assessment of Domino Accidents}

Khan and Abbasi ${ }^{[20]}$ presented a new computer-automated tool DOMIFFECT (DOMIno eFFECT) which is the first ever such tool reported for studying domino effect. Moreover, Khan and Abbasi ${ }^{[32]}$ proposed a systematic methodology called 'domino effect analysis' (DEA). 
Furthermore, they evolved a 'DEA procedure' and demonstrated its application to several reallife situations ${ }^{[33-35]}$. The study of DEA has two levels. The first is to identify the units within the context of specific industrial lay-out, which are likely to meet with an accident that has the potential to endanger one or more other units. The second is to assess consequences of the likely accidents, including the probability of the accident in the danger unit, which can lead to further accidents.

In view of DEA, the computer-automated tool DOMIFFECT had also been developed. Because of limited computational resources, it is complex for the application of the methodology to really industrial facilities. The development of quantitative risk assessment (QRA) methodologies is devoted to improve quantitative analysis of domino scenarios.

As a tool to provide quantitative information on the risk caused by conventional accidents in chemical and process plants, QRA intends to calculation of risk expressed by risk indexes such as individual risk and societal risk in risk assessment of industrial facilities ${ }^{[36-38]}$. QRA is consisted by a set of methodologies for estimating the risk posed by a given system in terms of human loss and economic loss. Although QRA is not an exact description of reality, it is a good, analytic predictive tool to assess the risk of complex process and storage facilities.

There are some research with QRA to estimate the risk of domino effect. Cozzani et al. ${ }^{[39]}$ developed a systematic procedure for quantitative assessment of the risk related to domino effect. Escalation vectors, defined as the physical effects responsible of possible accident propagation, were identified for the primary scenarios usually considered in the QRA procedure. Well-assessed procedures for quantitative evaluation of risk due to domino effect are still lacking. Based on recent advances in the modeling of fire and explosion damage to process equipment due to different escalation vectors (heat radiation, overpressure and fragment projection), Cozzani et al. ${ }^{[40]}$ focused on the adjustment and on the improvement of criteria for escalation credibility. Revised threshold values were proposed, and specific escalation criteria were obtained for the primary scenarios more frequently considered in the risk assessment of industrial sites. Bernechea et al. ${ }^{[41]}$ developed a simple method to include domino effect in QRAs of storage facilities, by estimating the frequency with which new accidents will occur due to domino effect. The results show that it can indeed be used to include the possibility of domino effect occurrence in a QRA. Bernechea and Arnaldos ${ }^{[42]}$ proposed a methodology that combines inherently safer design (ISD) strategies with quantitative risk assessment (QRA) to optimize the design of storage installations. The proposed method applies QRA to estimate the risk associated with a specific design. The design can then be compared to others to determine which is inherently safer. Al-shanini et al. ${ }^{[43]}$ presented a review of accident models that had been developed for the chemical process industry with in-depth analyses of a class of models known as dynamic sequential accident models (DSAMs).

With the QRA framework, some methods and models become available to allow quantitative assessment of domino accidents supported by specific software tools with geographic information systems. Cozzani et al. ${ }^{[44]}$ interfaced a geographical information system (GIS) platform to domino assessment software by a systematic methodology for identification of domino scenarios and for assessment of consequences and expected frequencies of escalation events. The GISbased software was a key element in the limitation of the effort required for the quantitative 
assessment of domino scenarios. On the basis of Cozzani et al. ${ }^{[4]}$, Antonioni et al. ${ }^{[45]}$ developed the Aripar-GIS software for risk recomposition. The results evidenced that quantitative risk assessment of escalation hazard was fundamental important for identifying critical equipment and addressing prevention and protection actions.

Some computer-automated tools have been developed for determining the probability of domino effect and assessing domino accidents in chemical processing industries ${ }^{[46-53]}$. For example, to estimate accidents involving toxic releases, explosions, and fires in chemical processing sites, Khan and Abbasi ${ }^{[46]}$ developed a computer-automated tool MAXCRED (MAXimum CREDible accident analysis). Reniers et al. ${ }^{[23]}$ proposed a software tool called DomPrevPlanning to prevent domino effect in a complex surrounding of chemical installations.

The four fundamental steps of QRA is identification, frequency assessment, consequence assessment and risk calculation/recomposition, and further details may be found in the literature ${ }^{[39]}$. Further, in order to analyze detailed procedure required for the quantitative assessment of risk due to domino scenarios, Cozzani et al. ${ }^{[39]}$ presented the basis for quantitative assessment of domino effects independent of the specific tools used to carry out any step of assessment. The framework of QRA may be applied to assess secondary or even higher level domino effect. Because of the complexity and computational resources required for the assessment increase exponentially with the level of the assessment, the method mainly focused on assessing the "first level" scenarios.

\subsubsection{Summary for Quantitative Risk Assessment}

The framework of QRA has recognized the first level of accidents where primary and secondary events are taking place, but neglected the higher levels of domino effect. Because higher-order events and synergistic effect of events of different orders are not considered in the modeling, the method results in an underestimation of the potential risk and leads to an improper allocation of safety measures. With the evolution pattern of domino accidents not be considered, the result is becoming the analysis of a cluster of accidents rather than a chain of accidents. The method calculates holistic probability of domino effect, neglecting the actual time line of the escalation process and the more likely time sequences of the domino scenario.

\subsection{Monte Carlo Simulation}

\subsubsection{Monte Carlo Simulation of Assessment of Domino Accidents}

"Monte Carlo Simulation (MCS)" was coined by Ulam and Metropolis in reference to games of chance, which is the main tourist attraction in Monte Carlo, Monaco. By using random numbers as inputs, MCS enables iterative evaluation of a system. The method is often used for highly complex, non-linear, or systems with many parameters. When potential probabilities of the process are known but their interaction is difficult to determine, MCS works especially well ${ }^{[54]}$.

As defined in the context of accidents in chemical process industry, the essence of domino effect phenomena makes itself accessed by MCS. There are two types of probabilities in estimation of domino frequency. The first type is primary accident probability, which is the probability of equipment playing a role as initiator in a chain of accidents. The primary accident probability 
can be gained by an event tree analysis or a fault tree analysis. Escalation probability is the second type of probability needed for domino effect analysis. The probability of this escalation can be estimated from probit models. The introduction of uncertain input parameters makes an analytical model become extremely complex with the complexity involved in estimating domino effect frequencies. Therefore, MCS can be selected among all other options to estimate domino effect frequencies.

A new model for assessing domino effect in process plants was presented recently by Abdolhamidzadeh et al. ${ }^{[4]}$. Monte Carlo simulation was applied to overcome the limitations of analytical methods in handling uncertainty and complexity associated with domino effect modeling. They proposed the FREEDOM (FREquency Estimation of DOMino accidents) based on conducting several hypothetical experiments to simulate the actual behavior of a multi-unit system which may undergo domino effect. Similar to those of other models, the model presented an algorithm and developed the sequences in a different way with a sophisticated mathematical tool. Ahmed et al. ${ }^{[55]}$ handled industrial accidents and domino effect that may occur in an industrial plant and performed sensitivity analysis by Monte Carlo simulations. The probability of impact and risk of failure of target tanks were reported.

The FREEDOM algorithm is defined as the combination of equipment present in an industrial unit that may or may not influence the failure of each other. A failure probability of each component of this system is the desired outcome. The FREEDOM algorithm is composed by two parts: inner and outer loops. The inner loop is selected according to failure rate of equipment, which is representative of average lifetime of the equipment. The outer loop operates for the iterations or experiments $N$ times. The steps associated with the operation of FREEDOM are: 1) Setting parameter input; 2) Initializing the experiment; 3) Checking for the initiating event; 4) Checking domino effect-I; 5) Checking domino effect-II; and 6) Keeping record. It can be seen that the time loop is stopped and a new iteration is started when all components are proved to have failed. This considerably reduces the run time of the program.

As stated by Abdolhamidzadeh et al. ${ }^{[4]}$, the algorithm is able to calculate failure probabilities of equipment even in situations which would be too complex for analytical methods. It is not constrained by how big or complex the system. The shortcoming of the FREEDOM is its inability to handle multiple failure scenarios. Rad et al. ${ }^{[56]}$ proposed a new method to assess the frequency of domino accidents called FREEDOM II, which overcame the limitation of FREEDOM and extended its capabilities. In the algorithm of FREEDOM II, a desired number of hypothetical random experiments were performed to determine the overall escalation probabilities, and the value of increased frequency for each scenario in a multi-unit system was calculated.

\subsubsection{Summary for Monte Carlo Simulation}

Compared to analytical methods, MCS has the following advantages. MCS provides a wide range of output parameters including different probability functions, while analytical methods are usually limited only to the expected values. The inherent advantages of MCS method is independent of complex system, while the model used in analytical techniques is usually a simplify system. 
According to the inherent advantages of the simulation technique-iterative evaluation of highly complex and non-linear systems using sets of random numbers as inputs, and the ability to handle more than just a few uncertain parameters, the FREEDOM (FREquency Estimation of DOMino accidents) has been exploited to develop an algorithm. The FREEDOM is able to handle a system, which can not be solvable by analytical techniques.

\subsection{Bayesian Networks}

\subsubsection{Bayesian Network for Domino Accidents Assessment}

Bayesian network $(\mathrm{BN})$ is a probabilistic graphical method for reasoning under uncertainty that has recently started to be used as a promising substitute for the majority of conventional methods in risk analysis and reliability engineering. BN is increasingly applied to reliability assessment, fault diagnosis, and constantly updated failure probability of safety systems. Some research have examined the parallels between BNs and fault tree (FT) and shown the obvious superiority of BNs over FTs in terms of modeling and analysis capabilities ${ }^{[57-61]}$. Other relevant work has been done by either mapping static FTs to BNs or mapping dynamic FTs into the corresponding dynamic $\mathrm{BNs}^{[58]}$. The next section will introduce the use of BNs to assess domino effect.

In $\mathrm{BN}$, the nodes represent variables and are connected by means of directed arcs. The arcs denote dependencies or causal relationships between the linked nodes. BN is a directed acyclic graph for reasoning under uncertainty. To model the likely propagation path of domino effect, the following steps are taken: 1) Identifying credible units; 2) Specifying primary unit; 3) Calculating escalation vectors; 4) Discriminating secondary units; 5) Distinguishing accident scenarios; and 6) Propagating domino effect to the next level. Synergistic effects should be considered when repeating the same procedure for either the secondary units or higher-order units.

According to Bayesian network, Khakzad et al. ${ }^{[28]}$ proposed a new methodology to model the propagation patterns of domino effect and to estimate domino effect probability at different levels. It is possible to analyze domino effect through a probabilistic framework, considering synergistic effects, noisy probabilities, and common cause failures by the flexible structure and the unique modeling techniques offered by Bayesian network. The probability of the domino effect at subsequent levels is accounted by the modified BN. Although many attempts have been made to identify the spatial evolution of domino effects, they overlooked the temporal evolution of such accidents.

As an extension of ordinary BN, Dynamic Bayesian network (DBN) promotes explicit modeling of temporal evolution of random variables over a discredited time line. DBN allows a node at $i$ th time slice to be conditionally dependent on its parents at the same time slice and its own states at previous time slices by dividing the time line to a number of time slices. Khakzad et al. ${ }^{[30]}$ proposed a methodology to model domino effect by the spatial and temporal evolution, and identified the sequence of accidents in a potential domino effect by dynamic Bayesian network. The developed methodology identified the most probable sequence of accidents by considering time dependencies. The methodology also performed backward analysis or probability updating through multiple observations which have been made at different times for a 
unit.

\subsubsection{Summary for Bayesian Network}

From both qualitative and quantitative points of view, BN is effectively suitable for the analysis of domino effect. From a qualitative perspective, with the flexible structure, BN can be applied to a wide range of accident scenarios and embed versatile types of information in the network by adding auxiliary nodes. By the means of nodes and causal arcs of BN, the graphical representation of units and escalation vectors can help to visualize the propagation pattern of domino effect. From a quantitative point of view, BN models different types of causal relationships among events by using the advantage of robust conditional probability tables (CPTs). With adding similar escalation vectors or using noisy probabilities in the case of different escalation vectors, CPTs can help in considering the synergistic effect of contributing events.

Taking DBN into consideration, the new methodology can study escalation of domino effect from both the spatial and temporal within chemical process plants. With taking time dependencies into account to identify the most probable sequence of accidents, the developed methodology reflects the characteristic of domino effect much better than the most probable combination of accidents offered by ordinary BN. The multiple observations made at different times for a unit can be incorporated into model to perform backward analysis or probability updating.

\section{Domino Effect Prevention in a Chemical Industrial Area}

\subsection{Game Theory for Domino Effect Prevention}

Game theory is a promising approach to address security issues ${ }^{[62-65]}$. Game theory model combined with reliability theory has been widely applied. The best balance between security and critical infrastructure investment costs is obtained by game-theoretical model ${ }^{[66,67]}$. Game theory is the theory of independent and interdependent decision making. Multi-person games of strategy are games involving three or more players, each of whom has partial control over the outcome. The external domino effect game can be classified as a two-enterprise mixed-motive game of strategy.

To prevent domino effect, Reniers et al. ${ }^{[68]}$ studied whether game theory was applicable to analyze external safety investment within a two-enterprise chemical cluster. The investment game that considered external domino effect of accidents was a mixed-motive game without a single equilibrium point. To analyze the behaviors of adjacent chemical enterprises and their negotiation on safety investment, Reniers and Soudan ${ }^{[9]}$ applied game-theoretic approach and provided a guide to enterprises to reach a win-win situation with regard to safety investment. There are more than two enterprises in a chemical cluster, so it is worth to analyze investment behavior of more enterprises. To analyze whether there was a possibility of changing choices of some enterprises that led the rest of enterprises to alter from a socially non-optimal situation to a socially optimal situation. Reniers et al. ${ }^{[10]}$ analyzed the investment behavior of three enterprises in a chemical cluster by using game theory. The choice of enterprise's investment strategy was a dynamic process. Yulia and Reniers ${ }^{[69]}$ considered a two-stage sequential move game be- 
tween adjacent chemical plants. They proposed a subsidy plan to improve cross-company safety management in a chemical cluster. To evaluate different strategic crossplant precaution collaboration situations, Reniers et al. ${ }^{[70]}$ presented multi-plant collaboration model by using game theory. With prevention management's perceptions and the information from different plants and data, the model intended to determine possible financial collaboration benefits within entire industrial area.

Because of existing many enterprises in a cluster, it is more realistic to study the behaviors of $N$ enterprises in the investment game. It is obvious that the effects of investment level on the accident risk should be considered, rather than just assuming that enterprises' accident probabilities are zero. In order to study the behavior of $N$-enterprise investment, Wu et al. ${ }^{[71]}$ presented an $N$-enterprise investment game to analyze investment strategies against the risk of domino effect of accidents in a chemical cluster. With different accident losses, both Nash equilibrium and Pareto equilibrium were gained. It can be found that system parameters such as the domino effect and cluster size have significant impact on the decision making behaviors of enterprises. Further, to analyze dynamic of investment selection and the adjustment of government to enterprise strategy selection, Wu et al. ${ }^{[72]}$ developed an $N$-enterprise investment evolutionary game to analyze the investment strategies against the risk of domino effect in a chemical cluster. The static game and evolutionary game were analyzed. Evolutionary stable strategies can be obtained through dynamic analysis. Further, the process of investment strategy selection was simulated and some conclusions were obtained. Enterprises take different investment strategies with the different parameters. The utility and proportion of high-level investment may increase with the addition of government subsidy. The utility and proportion of low-level investment decreases with the increasing of government penalty. The enterprises tend to take high-level investment with the addition of accident loss. Government regulated the investment behavior of enterprises through subsidy and penalty, prompting enterprises to choose a high-level investment to achieve the maximization of social utility.

\subsection{Summary for Domino Effect Prevention}

From the analysis of domino accidents prevention by using game theory, we can obtain that the research of domino accidents focus on static game, dynamic game and evolutionary game. Supposing an enterprise has limited knowledge about other enterprises' strategies, it adopts its cross-plant prevention decisions on their own preferences and the perceptions they have about the preferences of other enterprises' prevention management. The prevention management is directly affected by the investment cost and the potential domino effect losses. In the cluster, the relationship between enterprises is very complex, so it is necessary to study the behaviors of enterprises and discuss more sophisticated real-life problems with game model.

\section{Conclusion}

The domino effect occurs in many major accidents, increasing significantly both their complexity and their final effects and consequences. Although in recent years the interest on it has increased, the research achievement is still less compared to other aspects of industrial accidents. This is the reason that its main features are still insufficiently known.

The objective of this work is to analyze and classify the study of domino effect on review- 
ing the scientific literature. In our work, we summarize the study of domino effect from three aspects: the statistical analysis of domino accidents in the chemical process industry, the evaluation of domino accidents and the prevention of domino accidents in chemical clusters using game theory. The statistical analysis of domino accidents in the chemical process industry analyzes the main features of the accidents in which the domino effect has taken place, e.g. the time and the location, the origin and causes of domino accidents. The quantitative assessment of domino accidents has primarily focused on quantitative risk assessment (QRA), Bayesian Networks (BN) and Monte Carlo simulation (MCS).

The main results and conclusions of this work are summarized to the following points:

1) The highest frequency of domino accidents is among 1971-1980 and 1981-1990, and the highest frequency of domino accidents is in the developed countries. Domino accidents are more occurred in fixed installation than transportation. Mechanical failure is the main cause of domino effect, followed by human error and external events.

2) The framework of QRA has recognized the first level of accidents where primary and secondary events are taking place, but neglected the higher levels of domino effect. In view of the inherent advantages of simulation technique, the FREEDOM (FREquency Estimation of DOMino accidents) is able to handle a system, which can not be solvable by analytical techniques. With the flexible structure, BN can be applied to a wide range of accident scenarios and embed versatile types of information in the network by adding auxiliary nodes.

3) The prevention of domino accidents in chemical clusters using game theory is seldom, and there is much space for improvement in enterprises' efforts to manage risk of domino accidents.

From the summary, we can know that Bayesian networks (BN) and Monte Carlo simulation (MCS) need further development. The prevention of domino accidents in chemical clusters using game theory is relatively small. Future work can be focused on the prevention of domino accidents with game theory.

\section{References}

[1] Reniers G, Cozzani V. Domino effects in the process industries: Modelling, prevention and managing. Newnes, 2013.

[2] Kadri F, Chatelet E. Domino effect analysis and assessment of industrial sites: A review of methodologies and software tools. International Journal of Computers and Distributed Systems, 2013, 2(III): 1-10.

[3] Delvosalle C, Fievez C, Benjelloun F. Development of a methodology for the identification of potential domino effects in "SEVESO" industries. Proceedings 9th International Symposium on Loss Prevention and Safety Promotion in the Process Industries, 1998, 3: 1252-1261.

[4] Abdolhamidzadeh B, Abbasi T, Rashtchian D, et al. A new method for assessing domino effect in chemical process industry. Journal of Hazardous Materials, 2010, 182(1): 416-426.

[5] Kourniotis S P, Kiranoudis C T, Markatos N C. Statistical analysis of domino chemical accidents. Journal of Hazardous Materials, 2000, 71(1): 239-252.

[6] Darbra R M, Palacios A, Casal J. Domino effect in chemical accidents: Main features and accident sequences. Journal of Hazardous Materials, 2010, 183(1): 565-573.

[7] Clini F, Darbra R M, Casal J. Historical analysis of accidents involving domino effect. Chemical Engineering, 2010, 19: 335-340.

[8] Salzano E, Cozzani V. The analysis of domino accidents triggered by vapor cloud explosions. Reliability Engineering \& System Safety, 2005, 90(2): 271-284.

[9] Reniers G, Soudan K. A game-theoretical approach for reciprocal security-related prevention investment decisions. Reliability Engineering \& System Safety, 2010, 95(1): 1-9. 
[10] Reniers G, Dullaert W, Karel S. Domino effects within a chemical cluster: A game-theoretical modeling approach by using Nash-equilibrium. Journal of Hazardous Materials, 2009, 167(1): 289-293.

[11] Abdolhamidzadeh B, Rashtchian D, Morshedi M. Statistical survey of domino past accidents. Proc. 8th World Congress of Chemical Engineering, Montreal, 2009.

[12] Abdolhamidzadeh B, Abbasi T, Rashtchian D, et al. Domino effect in process-industry-an inventory of past events and identification of some patterns. Journal of Loss Prevention in the Process Industries, 2011, 24(5): 575-593.

[13] Chen Y, Zhang M, Guo P, et al. Investigation and analysis of historical domino effects statistic. Procedia Engineering, 2012, 45: 152-158.

[14] Oggero A, Darbra R M, Munoz M, et al. A survey of accidents occurring during the transport of hazardous substances by road and rail. Journal of Hazardous Materials, 2006, 133(1): 1-7.

[15] Hemmatian B, Abdolhamidzadeh B, Darbra R M, et al. The significance of domino effect in chemical accidents. Journal of Loss Prevention in the Process Industries, 2014, 29: 30-38.

[16] Ronza A, Félez S, Darbra R M, et al. Predicting the frequency of accidents in port areas by developing event trees from historical analysis. Journal of Loss Prevention in the Process Industries, 2003, 16(6): 551-560.

[17] Gomez-Mares M, Zárate L, Casal J. Jet fires and the domino effect. Fire Safety Journal, 2008, 43(8): 583-588.

[18] Zhang H D, Zheng X P. Characteristics of hazardous chemical accidents in China: A statistical investigation. Journal of Loss Prevention in the Process Industries, 2012, 25(4): 686-693.

[19] Khan F I, Abbasi S A. DOMIFFECT (DOMIno eFFECT): User-friendly software for domino effect analysis. Environmental Modelling \& Software, 1998, 13(2): 163-177.

[20] Khan F I, Abbasi S A. Models for domino effect analysis in chemical process industries. Process Safety Progress, 1998, 17(2): 107-123.

[21] Cozzani V, Salzano E. The quantitative assessment of domino effects caused by overpressure: Part I. Probit models. Journal of Hazardous Materials, 2004, 107(3): 67-80.

[22] Reniers G L L, Dullaert W, Ale B J M, et al. The use of current risk analysis tools evaluated towards preventing external domino accidents. Journal of Loss Prevention in the Process Industries, 2005, 18(3): $119-126$.

[23] Reniers G L L, Dullaert W. DomPrevPlanning: User-friendly software for planning domino effects prevention. Safety Science, 2007, 45(10): 1060-1081.

[24] Reniers G L L, Dullaert W. Knock-on accident prevention in a chemical cluster. Expert Systems with Applications, 2008, 34(1): 42-49.

[25] Nguyen Q B, Mebarki A, Saada R A, et al. Integrated probabilistic framework for domino effect and risk analysis. Advances in Engineering Software, 2009, 40(9): 892-901.

[26] Khakzad N, Khan F, Amyotte P. Dynamic safety analysis of process systems by mapping bow-tie into Bayesian network. Process Safety and Environmental Protection, 2013, 91(1): 46-53.

[27] Khakzad N, Khan F, Amyotte P. Risk-based design of process systems using discrete-time Bayesian networks. Reliability Engineering \& System Safety, 2013, 109: 5-17.

[28] Khakzad N, Khan F, Amyotte P, et al. Domino effect analysis using Bayesian networks. Risk Analysis, 2013, 33(2): 292-306.

[29] Khakzad N, Khan F, Amyotte P, et al. Risk management of domino effects considering dynamic consequence analysis. Risk Analysis, 2014, 34(6): 1128-1138.

[30] Khakzad N. Application of dynamic Bayesian network to risk analysis of domino effects in chemical infrastructures. Reliability Engineering \& System Safety, 2015, 138: 263-272.

[31] López-Molina A, Vázquez-Román R, Mannan M S, et al. An approach for domino effect reduction based on optimal layouts. Journal of Loss Prevention in the Process Industries, 2013, 26(5): 887-894.

[32] Khan F I, Abbasi S A. An assessment of the likelihood of occurrence, and the damage potential of domino effect (chain of accidents) in a typical cluster of industries. Journal of Loss Prevention in the Process Industries, 2001, 14(4): 283-306.

[33] Khan F I, Natarajan B R, Abbasi S A. Avoid the domino effect via proper risk assessment. Chemical Engineering Progress, 2000, 96(10): 63-72.

[34] Khan F I, Abbasi S A. Estimation of probabilities and likely consequences of a chain of accidents (domino effect) in Manali Industrial Complex. Journal of Cleaner Production, 2001, 9(6): 493-508. 
[35] Khan F I, Husain T, Abbasi S A. Safety weighted hazard index (SWeHI): A new, user-friendly tool for swift yet comprehensive hazard identification and safety evaluation in chemical process industries. Process Safety and Environmental Protection, 2001, 79(2): 65-80.

[36] Baesi S, Abdolhamidzadeh B, Hassan C R C, et al. Application of a multi-plant QRA: A case study investigating the risk impact of the construction of a new plant on an existing chemical plant's risk levels. Journal of Loss Prevention in the Process Industries, 2013, 26(5): 895-903.

[37] Nomen R, Sempere J, Mariotti V. QRA including domino effect as a tool for engineering design. Procedia Engineering, 2014, 84: 23-32.

[38] Cozzani V, Antonioni G, Landucci G, et al. Quantitative assessment of domino and NaTech scenarios in complex industrial areas. Journal of Loss Prevention in the Process Industries, 2014, 28: 10-22.

[39] Cozzani V, Gubinelli G, Antonioni G, et al. The assessment of risk caused by domino effect in quantitative area risk analysis. Journal of Hazardous Materials, 2005, 127(1): 14-30.

[40] Cozzani V, Gubinelli G, Salzano E. Escalation thresholds in the assessment of domino accidental events. Journal of Hazardous Materials, 2006, 129(1): 1-21.

[41] Bernechea E J, Vílchez J A, Arnaldos J. A model for estimating the impact of the domino effect on accident frequencies in quantitative risk assessments of storage facilities. Process Safety and Environmental Protection, 2013, 91(6): 423-437.

[42] Bernechea E J, Arnaldos J. Optimizing the design of storage facilities through the application of ISD and QRA. Process Safety and Environmental Protection, 2014, 92(6): 598-615.

[43] Al-shanini A, Ahmad A, Khan F. Accident modelling and analysis in process industries. Journal of Loss Prevention in the Process Industries, 2014, 32: 319-334.

[44] Cozzani V, Antonioni G, Spadoni G. Quantitative assessment of domino scenarios by a GIS-based software tool. Journal of Loss Prevention in the Process Industries, 2006, 19(5): 463-477.

[45] Antonioni G, Spadoni G, Cozzani V. Application of domino effect quantitative risk assessment to an extended industrial area. Journal of Loss Prevention in the Process Industries, 2009, 22(5): 614-624.

[46] Khan F I, Abbasi S A. MAXCRED - A new software package for rapid risk assessment in chemical process industries. Environmental Modelling \& Software, 1998, 14(1): 11-25.

[47] Khan F I, Abbasi S A. Risk analysis of a chloralkali industry situated in a populated area using the software package MAXCRED-II. Process Safety Progress, 1997, 16(3): 172-184.

[48] Egidi D, Foraboschi F P, Spadoni G, et al. The ARIPAR project: Analysis of the major accident risks connected with industrial and transportation activities in the Ravenna area. Reliability Engineering \& System Safety, 1995, 49(1): 75-89.

[49] Spadoni G, Contini S, Uguccioni G. The new version of ARIPAR and the benefits given in assessing and managing major risks in industrialised areas. Process Safety and Environmental Protection, 2003, 81(1): 19-30.

[50] Ditali S, Colombi M, Moreschini G, et al. Consequence analysis in LPG installation using an integrated computer package. Journal of Hazardous Materials, 2000, 71(1): 159-177.

[51] Cozzani V, Tugnoli A, Salzano E. Prevention of domino effect: From active and passive strategies to inherently safer design. Journal of Hazardous Materials, 2007, 139(2): 209-219.

[52] Tixier J, Dusserre G, Rault-Doumax S, et al. OSIRIS: Software for the consequence evaluation of transportation of dangerous goods accidents. Environmental Modelling \& Software, 2002, 17(7): 627-637.

[53] Lee J Y, Lee J W, Ko J, et al. Optimization for allocating the explosive facilities in order to minimize the domino effect using nonlinear programming. Korean Journal of Chemical Engineering, 2005, 22(5): 649-656.

[54] Farmer F R. Reliability evaluation of engineering systems: Concepts and techniques. Discrete Applied Mathematics, 2014, 8(2): 213-214.

[55] Ahmed M, Jerez S, Matasic I, et al. Explosions and structural fragments as industrial hazard: Domino effect and risks. Procedia Engineering, 2012, 45: 159-166.

[56] Rad A, Abdolhamidzadeh B, Abbasi T, et al. FREEDOM II: An improved methodology to assess domino effect frequency using simulation techniques. Process Safety and Environmental Protection, 2014, 92(6): $714-722$.

[57] Marquez D, Neil M, Fenton N. Improved reliability modeling using Bayesian networks and dynamic discretization. Reliability Engineering \& System Safety, 2010, 95(4): 412-425. 
[58] Khakzad N, Khan F, Amyotte P. Safety analysis in process facilities: Comparison of fault tree and Bayesian network approaches. Reliability Engineering \& System Safety, 2011, 96(8): 925-932.

[59] Matellini D B, Wall A D, Jenkinson I D, et al. Modelling dwelling fire development and occupancy escape using Bayesian network. Reliability Engineering \& System Safety, 2013, 114: 75-91.

[60] Pasman H, Rogers W. Bayesian networks make LOPA more effective, QRA more transparent and flexible, and thus safety more definable. Journal of Loss Prevention in the Process Industries, 2013, 26(3): 434-442.

[61] Weber P, Medina-Oliva G, Simon C, et al. Overview on Bayesian networks applications for dependability, risk analysis and maintenance areas. Engineering Applications of Artificial Intelligence, 2012, 25(4): 671682 .

[62] Liu D, Wang X F, Camp J. Game-theoretic modeling and analysis of insider threats. International Journal of Critical Infrastructure Protection, 2008, 1: 75-80.

[63] Golany B, Kaplan E H, Marmur A, et al. Nature plays with dice-terrorists do not: Allocating resources to counter strategic versus probabilistic risks. European Journal of Operational Research, 2009, 192(1): 198-208.

[64] Hausken K, Levitin G. Minmax defense strategy for complex multi-state systems. Reliability Engineering \& System Safety, 2009, 94(2): 577-587.

[65] Shafi K, Bender A, Zhong W, et al. Spatio-temporal dynamics of security investments in an interdependent risk environment. Physica A: Statistical Mechanics and Its Applications, 2012, 391(20): 5004-5017.

[66] Bier V M, Nagaraj A, Abhichandani V. Protection of simple series and parallel systems with components of different values. Reliability Engineering \& System Safety, 2005, 87(3): 315-323.

[67] Azaiez M N, Bier V M. Optimal resource allocation for security in reliability systems. European Journal of Operational Research, 2007, 181(2): 773-786.

[68] Reniers G. An external domino effects investment approach to improve cross-plant safety within chemical clusters. Journal of Hazardous Materials, 2010, 177(1): 167-174.

[69] Yulia P, Reniers G. A sequential-move game for enhancing safety and security cooperation within chemical clusters. Journal of Hazardous Materials, 2011, 186(1): 401-406.

[70] Reniers G, Cuypers S, Pavlova Y. A game-theory based multi-plant collaboration model (MCM) for crossplant prevention in a chemical cluster. Journal of Hazardous Materials, 2012, 209: 164-176.

[71] Wu J, Yang H, Cheng Y, et al. An $N$-enterprise investment game against the risk of domino accidents in a chemical cluster: Nash and Pareto equilibria. Working paper, 2015.

[72] Wu J, Yang H, Cheng Y, et al. An evolutionary game approach to developing an $N$-enterprise investment strategy for domino accident prevention in a chemical cluster. Working paper, 2015. 